\title{
EFECTO DE LAS SEMILLAS DE MORINGA (Moringa oleifera Lam.) EN LAS CONDICIONES PARA LA CLARIFICACIÓN DEL AGUA DEL RÍO SAMA
}

\author{
Marcial Alfredo Castillo Cohaila*a, Edgardo Óscar Avendaño Cáceres ${ }^{\mathrm{b}}$
}

\begin{abstract}
RESUMEN
En el tratamiento de clarificación del agua del agua del río Sama con semillas de moringa de La Yarada Los Palos se logró una eficiente remoción de turbidez como consecuencia de la eliminación de las partículas en suspensión contenidas en el agua del río Sama en época de lluvias. Por lo manifestado el objetivo de la investigación fue determinar las condiciones para el tratamiento con semillas de moringa en la clarificación del agua del río Sama empleando el método de prueba de jarras con dosis de semillas de moringa de 0,1 y $0,2 \mathrm{~g} / \mathrm{L}$; velocidad de floculación de 20 y 30 rpm y tiempo de floculación de 20 y 30 minutos. Las mejores condiciones de operación requeridas para el tratamiento del agua fueron: dosis de semillas de moringa (agente coagulante) $0,2 \mathrm{~g} / \mathrm{L}$, velocidad de floculación 30 revoluciones por minuto (rpm) y un tiempo de floculación de 30 minutos. El tiempo de sedimentación de 90 minutos se estableció con agua del río Sama con niveles de turbidez de 132, 48,6 y 424 unidades nefelométricas de turbiedad (UNT). La semilla de moringa fue de la producción del año 2018 proveniente del distrito de La Yarada Los Palos, con la cual se obtuvo una composición proximal de 5,70 \% de humedad (Método AOAC), 30,78\% de proteínas (Método micro Kjeldahl), 40,36\% de materia grasa (Método Soxhlet), 4,42 \% de cenizas (Método AOAC) y 18,74 \% de carbohidratos (por diferencia). Se utilizaron los diseños experimentales $2^{3}$ y el diseño de bloques completos al azar. Se obtuvo niveles de reducción de la turbidez de 97,04 \%; 92,37 \% y 98,88 \%.
\end{abstract}

Palabras clave: semillas de moringa, turbidez, clarificación, prueba de jarras.

\footnotetext{
a Facultad de Ciencias Agropecuarias, Universidad Nacional Jorge Basadre Grohmann, Av. Miraflores s/n, Tacna, Perú, mcastilloc@unjbg.edu.pe

${ }^{\text {b }}$ Facultad de Ingeniería, Universidad Nacional Jorge Basadre Grohmann.
} 


\title{
EFFECT OF MORINGA SEEDS (Moringa oleifera Lam.) ON THE CONDITIONS FOR CLARIFICATION OF WATER FROM THE SAMA RIVER OF TACNA
}

\begin{abstract}
In the clarification treatment of the water from the Sama river water with moringa seeds from La Yarada Los Palos, an efficient removal of turbidity was achieved as a consequence of the removal of the suspended particles contained in the water of the Sama river in the rainy season. Therefore, the objective of the investigation was to determine the conditions for the treatment with moringa seeds in the clarification of the water of the Sama river using the test method of jugs with moringa seeds doses of 0,1 and $0,2 \mathrm{~g} / \mathrm{L}$; flocculation speed of 20 and 30 rpm and flocculation time of 20 and 30 minutes. The best operating conditions required for water treatment were: moringa seeds doses (coagulating agent) $0,2 \mathrm{~g} / \mathrm{L}$, flocculation speed 30 revolutions per minute $(\mathrm{rpm})$ and a flocculation time of 30 minutes. Settling time of 90 minutes was established with water from the Sama River with turbidity levels of $132 ; 48,6$ and 424 nephelometric turbidity units (UNT). Moringa seed was from the production of the year 2018 from the district of La Yarada Los Palos, with which a proximal composition of 5,70\% Humidity was obtained (AOAC Method), 30,78\% protein (Micro Kjeldahl Method ), 40,36\% fat (Soxhlet Method), 4,42\% ash (AOAC Method) and 18,74\% carbohydrates (by difference). The experimental designs $2^{3}$ and the randomized complete block design were used. Turbidity reduction levels of $97,04 \%$ were obtained; $92,37 \%$ and $98,88 \%$.
\end{abstract}

Key words: moringa seeds, turbidity, clarification, jar test.

\section{INTRODUCCIÓN}

La orientación a la utilización de elementos naturales para el tratamiento del agua ${ }^{1}$ sugiere vigilar y controlar la presencia de aluminio en el agua potable ${ }^{2}$ y romper el paradigma de su utilización ${ }^{3}$ que como en el caso de la semilla de moringa, representen un eficiente y seguro coagulante en el proceso de purificación del agua considerando sus propiedades floculantes $^{5}$ y su eficiencia comprobada ${ }^{6,7,8}$ y justificando su uso por ser una alternativa ambientalmente correcta $^{9}$. Además ${ }^{10}$, después de ensayar diversos tipos de clarificadores tradicionales, comprobó que las semillas de moringa oleífera son las más efectivas como coagulantes, siendo su acción comparable al sulfato de aluminio además de eliminar un elevado porcentaje de bacterias y de no tener acción tóxica.

La efectividad de las semillas de moringa para la remoción de materias en suspensión en aguas turbias ${ }^{11}$, constituye una efectiva alternativa de biorremediación ${ }^{12}$ constituyendo un excelente insumo para el tratamiento de la turbidez del agua en zonas rurales y en general para los países en vías de desarrollo ${ }^{4}$ permitiendo reducir drásticamente el número de partículas suspendidas $^{13}$, logrando ${ }^{14}$ remover turbiedades superiores a $95 \%$. 


\section{PARTE EXPERIMENTAL}

La investigación se realizó en las instalaciones de la Universidad Nacional Jorge Basadre Grohmann de Tacna, en el Laboratorio de Procesos Químicos de la Escuela Profesional de Ingeniería Química, en el Laboratorio Central de la Escuela Profesional de Agronomía y en el Laboratorio de Análisis de Alimentos de la Escuela Profesional de Ingeniería en Industrias Alimentarias y consistió en determinar mediante un diseño de experimento factorial $2^{3}$ las condiciones de los factores: dosis de semilla, velocidad de floculación y tiempo de floculación; complementando finalmente con un diseño experimental de bloques completos al azar para establecer el tiempo de sedimentación.

\section{Diseño de experimento factorial $2^{3}$}

Los factores con los que se realizaron los tratamientos fueron dosis de semillas de moringa de 0,1 y 0,2 g/L, velocidad de floculación de 20 y $30 \mathrm{rpm}$ y tiempo de floculación de 20 y 30 minutos.

\section{Diseño de bloques completos al azar}

Para determinar el tiempo de sedimentación, se realizaron cuatro tratamientos con tiempos de 1 hora, $1,5 \mathrm{~h}, 2 \mathrm{~h}$ y $2,5 \mathrm{~h}$. Los bloques establecidos por la turbiedad del agua del río Sama fueron a 48,6 UNT, 132 UNT y 424 UNT.

\section{Procedimiento y métodos para el tratamiento del agua}

El procedimiento para el tratamiento del agua, inició con el muestreo de agua del río Sama, para la realización de cada réplica del experimento, seguidamente se caracterizó la turbidez del agua recolectada, se sometió a tratamiento con harina de semillas de moringa (como coagulante), se analizó la turbidez del agua clarificada y se determinó el efecto de los tratamientos.

Previo al tratamiento del agua cruda recolectada del río Sama, se realizó su decantación durante 18 horas (registrándose 154 UNT) para el diseño de experimento factorial $2^{3}$; y para el diseño experimental de bloques completos al azar: 6 horas para el bloque 3, 20 horas para el bloque 1; y para el bloque 2 se combinó parte de las aguas de los bloques 1 y 3 , obteniéndose los valores de turbidez indicados en diseño de bloques completos al azar.

Todos los tratamientos en el equipo probador de jarras (Jar Test) JLT6 marca VELP Scientifica iniciaron con una velocidad de agitación rápida de $200 \mathrm{rpm}$, utilizando un volumen de agua del río Sama de 1 litro en cada tratamiento.

El efecto de los tratamientos (dosis de harina de semillas de moringa, velocidad de floculación y tiempo de floculación) en el agua del río Sama, se evaluó mediante la turbidez con un turbidímetro marca HACH modelo 2 100Q con rango de medida de 0 a 1000 UNT. 


\section{Procedimiento y métodos para las semillas}

Consistió en cinco etapas: recolección de las vainas de moringa, obtención de las semillas de moringa, caracterización física de las semillas, acondicionamiento de las semillas, determinación proximal de las semillas.

Para la obtención de la harina de semillas de moringa se procedió a retirar manualmente las vainas de la moringa, de una a la vez, para lo cual se tomó cada vaina de moringa con una mano y se procedió a abrirla tirando en sentido opuesto las manos y colectando las semillas en una jaba plástica desde donde se trasladó a una bolsa plástica para su almacenamiento. La cantidad total de semillas colectadas fue de 810 gramos.

La caracterización física de las semillas se realizó mediante el método mencionado por Belén et al., (2005), que consistió en tomar al azar semillas de moringa, retirarles la cáscara que cubre al endospermo utilizándose la parte del mango de madera de un cuchillo de cocina con el que se golpeó la semilla debilitándose la cubierta que fue separada y fragmentada manualmente determinándose sus pesos promedio.

Para el acondicionamiento de las semillas se procedió a triturar las semillas en una licuadora doméstica para granos marca National hasta reducir su tamaño a polvo, el cual, posteriormente se tamizó con una criba W.S. TYLER número 35 (32 Mesh) hasta obtener el producto sin partículas mayores al tamaño de malla. Finalmente, se pesaron 10 gramos de harina de moringa requerida para adicionarlos a $100 \mathrm{~mL}$ de agua destilada donde se mezcló por un tiempo de 5 minutos, después se realizó un filtrado rápido para separar las partículas de harina de moringa de la solución, la concentración de la solución fue de $100 \mathrm{~g}$ de harina de moringa/L de agua destilada, con niveles de turbidez de 139 UNT en promedio.

La determinación proximal de las semillas se realizó utilizándose una estufa marca Memmert para la determinación de humedad por el método de secado de la American Officials Methods Analytical (A.O.A.C) 925.10 de la 18th Edition 2005, para el contenido de materia grasa se utilizó el equipo Soxhlet marca Labconco por el método A.O.C.S Aa4-38, 1993, proteínas por el método A.O.A.C 920.87 de la 18th Edition 2005, utilizándose el equipo Kjeldahl marca Labconco y cenizas totales por el método de A.O.A.C 923.03 de la 18th Edition 2005, utilizándose una mufla marca Merkel con rango de temperatura de 0 a $800{ }^{\circ} \mathrm{C}$.

\section{Procesamiento y análisis de datos}

Para el procesamiento de los datos se utilizó los siguientes softwares: Excel, Statgraphics Versión XVI y Minitab Versión 18. El análisis de datos con el diseño de experimento factorial $2^{3}$, se realizó a los resultados de las mediciones de turbidez del agua del río Sama. Las pruebas de hipótesis realizadas fueron mediante el análisis de varianza con $95 \%$ de confianza comprobándose los supuestos del ANOVA y determinándose la significancia de los tres factores experimentales. El factor tiempo de sedimentación se analizó mediante un ANOVA aplicado al diseño de bloques completamente al azar con $95 \%$ de confianza. La diferencia entre los tratamientos se evalúo con la prueba de Tukey con $95 \%$ de confianza. 


\section{RESULTADOS Y DISCUSIÓN}

\section{Composición proximal}

Los dos principales componentes de las semillas de moringa de La Yarada Los Palos son la grasa con 40,36\% y las proteínas con $30,78 \%$ (tabla 1 ).

Tabla 3. Resultados de MR en muestras comerciales de té verde y café

\begin{tabular}{lc}
\hline Descripción & Cantidad (\%) \\
\hline Humedad & 5,70 \\
Grasa & 40,36 \\
Proteínas & 30,78 \\
Cenizas & 4,42 \\
Carbohidratos & 18,74 \\
\hline
\end{tabular}

La composición proximal de las semillas de moringa utilizada en la investigación es similar a las semillas de moringa procedentes del noroeste de la frontera de Pakistán, e inferior a lo determinado en la región de $\operatorname{Sindh}^{15}$; el contenido de grasa de 40,36 \% se encuentra entre los promedios de las dos regiones de Pakistán, el contenido de proteínas de 30,78 \% también se encuentra entre los promedios de las dos regiones; y el contenido de cenizas de 4,42\% de la investigación es menor a lo determinado por los investigadores mencionados, estimándose que esta diferencia se debería a la utilización integral de las semillas de moringa de parte de los investigadores mencionados, que conllevó al incremento de las cenizas en sus resultados.

\section{Turbidez con tratamientos con diseño $2^{3}$}

La dosis de semillas de moringa de La Yarada Los Palos utilizada para el tratamiento del agua del río Sama fue de 0,1 a 0,2 g/L; la velocidad de floculación y el tiempo de floculación de los tratamientos fueron de $20 \mathrm{rpm}$ y 30 minutos, respectivamente. El factor tiempo de sedimentación que no fue parte del diseño fue para todos los tratamientos 60 minutos, obteniéndose valores de turbidez mínimos de 7,07 y 7,1 UNT en cada réplica ( 1 y 2) de los tratamientos realizados (tabla 2).

Con el diseño de experimento factorial $2^{3}$, los 3 factores, dosis de semilla de moringa, velocidad de floculación y tiempo de floculación resultaron significativos, siendo el factor tiempo de floculación el de menor efecto, mientras que las interacciones entre los factores fueron significativas excepto para la interacción entre la dosis de semilla y tiempo de floculación para la turbidez del agua del río Sama (figura 1). 
Tabla 2. Turbidez del agua a diferentes tratamientos con semillas de moringa de La Yarada Los Palos, según diseño de experimento factorial $2^{3}$.

\begin{tabular}{ccccc}
\hline $\begin{array}{c}\text { Dosis de } \\
\text { semillas }\end{array}$ & $\begin{array}{c}\text { Velocidad de } \\
\text { floculación }\end{array}$ & $\begin{array}{c}\text { Tiempo de } \\
\text { floculación }\end{array}$ & \multicolumn{2}{c}{ Turbidez (UNT) } \\
\hline$(\mathrm{g} / \mathrm{L})$ & $(\mathrm{rpm})$ & $(\mathrm{min})$ & 1 & 2 \\
\hline 0,1 & 20 & 20 & 14,3 & 14,1 \\
0,2 & 20 & 20 & 9,11 & 9,3 \\
0,1 & 20 & 30 & 15,6 & 17 \\
0,2 & 20 & 30 & 11,3 & 10,5 \\
0,1 & 30 & 20 & 10,7 & 11,3 \\
0,2 & 30 & 20 & 10,2 & 9,8 \\
0,1 & 30 & 30 & 6,96 & 6,6 \\
0,2 & 30 & 30 & 7,07 & 7,1 \\
\hline
\end{tabular}

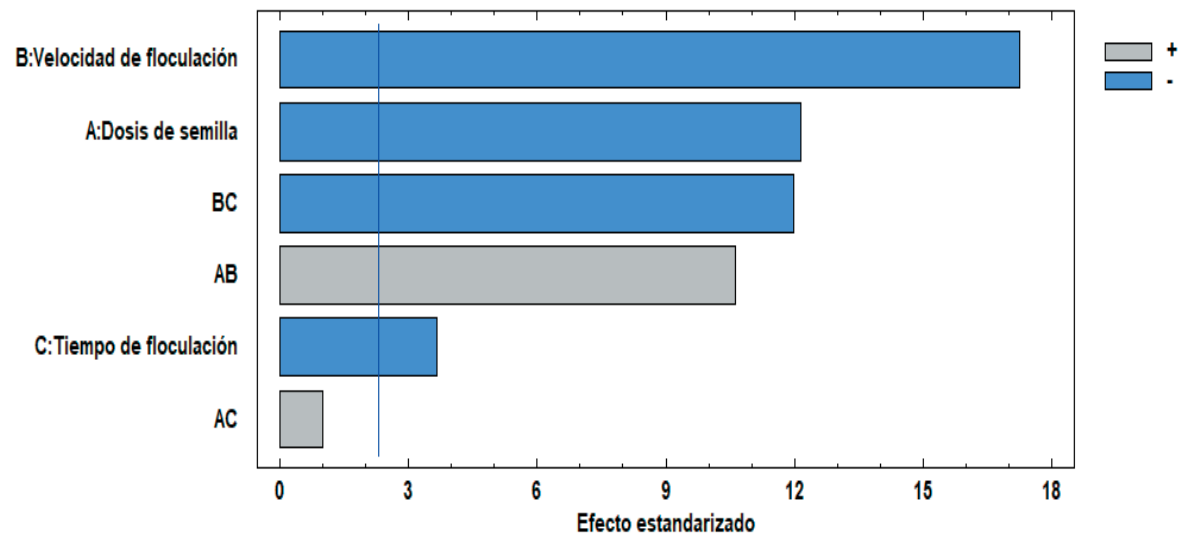

Figura 1. Significancia de los factores en la reducción de la turbidez del agua del río Sama por tratamiento con semillas de moringa proveniente de La Yarada Los Palos según diseño de experimento factorial $2^{3}$. 
La turbidez del agua del río Sama es reducida al máximo cuando se utiliza dosis de semilla de moringa de $0,2 \mathrm{~g} / \mathrm{L}$, velocidad de floculación de $30 \mathrm{rpm}$ y tiempo de floculación de 30 minutos (figura 2).za.

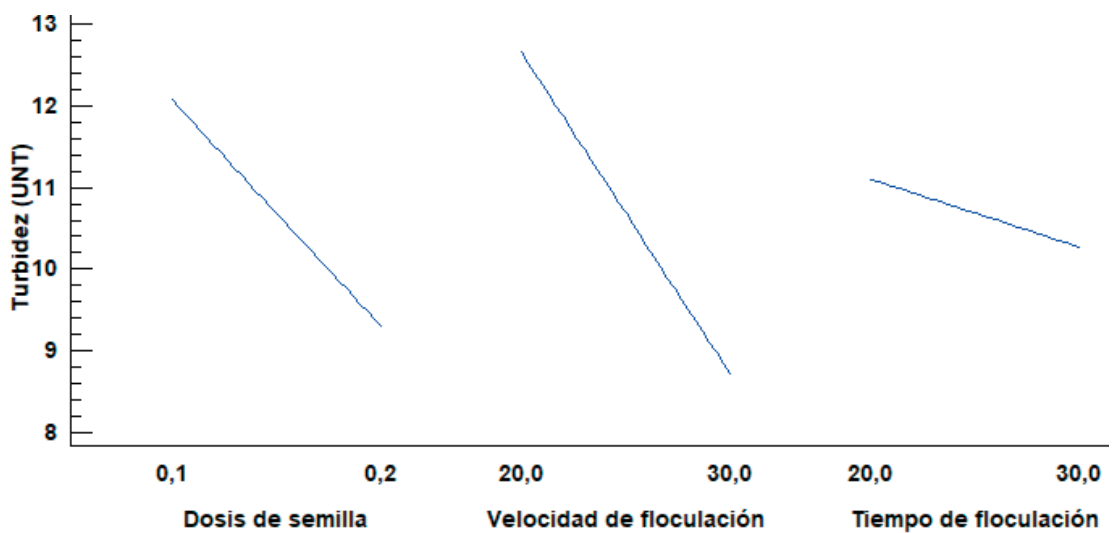

Figura 2. Efecto de la dosis de semillas, velocidad de floculación y tiempo de floculación en la reducción de turbidez del agua del río Sama por tratamiento con semilla de moringa de La Yarada Los Palos con diseño de experimento factorial $2^{3}$.

La reducción de la turbidez del agua del río Sama es mayor conforme se incrementa la dosis de semillas desde 0,1 a $0,2 \mathrm{~g} / \mathrm{L}$; así con una turbidez inicial del agua de 48,6 UNT y con 0,2 $\mathrm{g} / \mathrm{L}$ de dosis de semillas de moringa, se alcanzó una reducción de 93,44\%; mientras que ${ }^{16}$ lograron reducir la turbidez del agua en un $64 \%$ utilizando $0,013 \mathrm{~g} / \mathrm{L}$ en el tratamiento del agua con $50 \mathrm{UNT}$.

En cuanto a la velocidad de floculación, utilizando semillas de moringa con una velocidad de floculación de $45 \mathrm{rpm}$, obtuvieron una remoción de la turbidez del agua de $95, \%{ }^{17}$, y con una velocidad de floculación de $50 \mathrm{rpm}$.

El mejor tiempo de floculación para la semilla de moringa proveniente de Tacna fue de 30 minutos; mientras empleando 25 minutos de tiempo de floculación utilizando diversos coagulantes, para la clarificación de aguas para consumo humano con una turbidez inicial de 40,8 UNT, utilizando coagulantes naturales entre los cuales estuvo la moringa, removieron $86,15 \%$ de turbiedad ${ }^{18}$.

\section{Tiempo de sedimentación}

El incremento del tiempo de sedimentación de 1 hora a 1,5;2 y 2,5 horas, en promedio reduce la turbidez del agua del río Sama significativamente a 5,50; 3,91; 3,39 y 3,17 UNT, respectivamente para agua con turbidez inicial de 132 UNT. El incremento del tiempo de 
sedimentación de 1 hora a 1,5; 2 y 2,5 horas, en promedio reduce la turbidez del agua del río Sama significativamente a 4,04; 3,71; 3,19 y 3,22 UNT, respectivamente para agua con turbidez inicial de 48,6 UNT. El incremento del tiempo de sedimentación de 1 hora a 1,5; 2 y 2,5 horas, en promedio reduce la turbidez del agua del río Sama significativamente a 7,0; 4,77; 3,55 y 3,26 UNT, respectivamente para agua con turbidez inicial de 424 UNT (figura 3 ).

Estos resultados del tiempo de sedimentación permiten establecer el cumplimiento de la norma de DIGESA (reglamento de agua para consumo humano) que establece que el valor límite para la turbiedad es de 5 UNT.

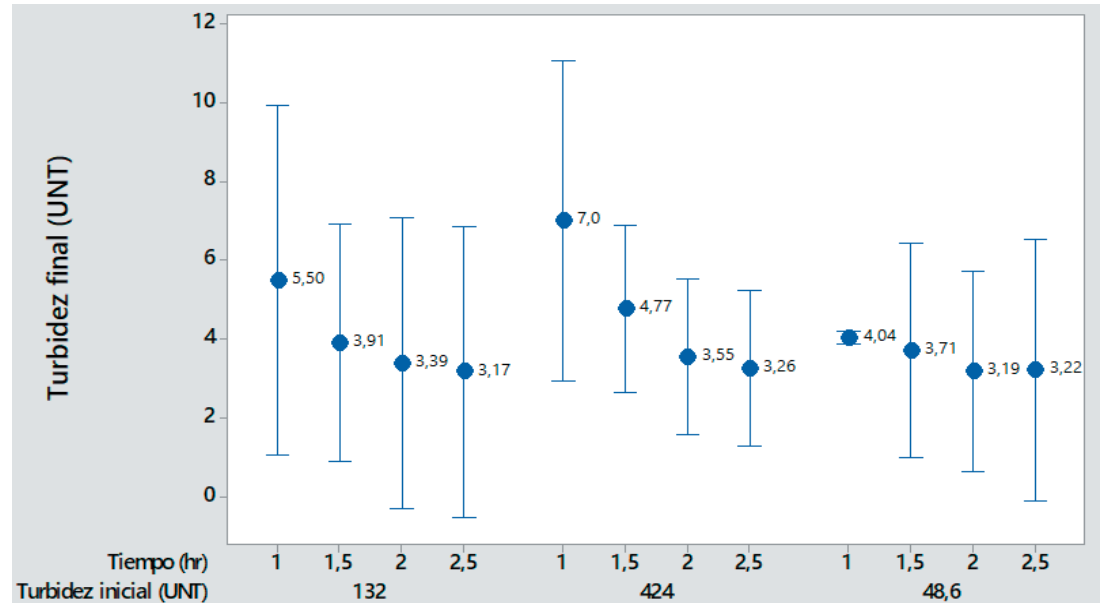

Figura 3. Turbidez del agua del río Sama tratada con semillas de moringa de La Yarada Los Palos en 3 diferentes muestras de agua y a 4 diferentes tiempos de sedimentación.

De los tres grupos homogéneos de muestras de agua del río Sama tratada para la reducción de la turbidez con semilla de moringa de La Yarada Los Palos, mediante la prueba de Tukey se determinó que con 1,5 horas de sedimentación se alcanza la mayor eliminación de la turbidez del agua del río Sama con $95 \%$ de confianza (tabla 3).

Tabla 3. Prueba de Tukey para la reducción de turbidez por tratamiento con semillas de moringa de La Yarada Los Palos durante la sedimentación del agua del río Sama.

\begin{tabular}{llllll}
\hline Tiempo & Casos & Media LS & Sigma LS & Grupos Homogéneos \\
\hline 2,5 & 6 & 3,22 & 0,25 & $\mathrm{X}$ & \\
2 & 6 & 3,38 & 0,25 & $\mathrm{X}$ & \\
1,5 & 6 & 4,13 & 0,25 & $\mathrm{X}$ & \\
1 & 6 & 5,51 & 0,25 & & $\mathrm{X}$ \\
\hline
\end{tabular}




\section{Porcentaje de reducción de la turbidez}

Con una turbidez del agua del río Sama de 132 UNT, después de una hora de sedimentación se reduce el 95,8 \% de la turbidez y a la hora y media se reduce el 97,04\%. Con una turbidez del agua del río Sama de 48,6 UNT, después de una hora de sedimentación se reduce el $90,14 \%$ de la turbidez y a la hora y media se reduce el $92,4 \%$. Con una turbidez del agua del río Sama de 424 UNT, después de una hora de sedimentación se reduce el 98,3\% de la turbidez y a la hora y media se reduce el 98,9\% (tabla 4); mientras que en otro estudio, empleando 60 minutos de tiempo de sedimentación, lograron una remoción de la turbidez de $95 \%$ empleando polvo de semilla Cassia fistula ${ }^{19}$.

Las condiciones para la reducción de la turbidez fueron, dosis de semilla de moringa $0,2 \mathrm{~g} / \mathrm{L}$, velocidad de floculación de $30 \mathrm{rpm}$ y tiempo de floculación de 30 minutos.

Tabla 4. Porcentaje de reducción de turbidez de tres calidades de agua del río Sama por tratamiento con semillas de moringa de La Yarada Los Palos.

\begin{tabular}{cccc}
\hline \multirow{2}{*}{$\begin{array}{c}\text { Tiempo } \\
\text { (hr) }\end{array}$} & \multicolumn{3}{c}{$\%$ de reducción de turbidez del agua } \\
\cline { 2 - 4 } & $132 \mathrm{UNT}$ & $48,6 \mathrm{UNT}$ & $424 \mathrm{UNT}$ \\
\hline 1 & $95,84 \%$ & $90,14 \%$ & $98,35 \%$ \\
1,5 & $97,04 \%$ & $92,37 \%$ & $98,88 \%$ \\
2 & $97,43 \%$ & $93,44 \%$ & $99,16 \%$ \\
2,5 & $97,60 \%$ & $93,37 \%$ & $99,23 \%$ \\
\hline
\end{tabular}

\section{CONCLUSIONES}

Con dosis de $0,2 \mathrm{~g} / \mathrm{L}$, velocidad de floculación de $30 \mathrm{rpm}$, tiempo de floculación de 30 minutos y tiempo de sedimentación de 90 minutos, las semillas de moringa, procedentes de la Yarada Los Palos, eliminan entre el 92,37 \% y 98,88 \% de la turbidez del agua del río Sama, esto conforme se incrementa la turbidez inicial del agua del río Sama desde 48,6 UNT hasta 424 UNT, respectivamente.

El incremento del tiempo de sedimentación de 1 hora a 1,5 horas, durante el tratamiento con semillas de moringa procedente de La Yarada Los Palos mejora el proceso de clarificación del agua del río Sama independientemente de los valores de su turbiedad inicial de 48,6; 132 y 424 UNT. 


\section{AGRADECIMIENTO}

Se agradece el apoyo de: la Escuela Profesional de Ingeniería Química de la Universidad Nacional Jorge Basadre Grohmann de Tacna por permitir el uso de las instalaciones y el equipamiento del laboratorio de procesos químicos, la Escuela Profesional de Agronomía por el uso del Laboratorio Central y la Escuela Profesional de Ingeniería en Industrias Alimentarias por el apoyo en el uso del Laboratorio de Análisis de Alimentos.

\section{REFERENCIAS BIBLIOGRÁFICAS}

1. Paredes P. El uso de Moringa oleifera como material natural para el tratamiento del agua potable en países en vía de desarrollo. Yachana. 2013; 2(2):243-246.

2. Trejo R, Hernández V. Riesgos a la salud por presencia del aluminio en el agua potable. Conciencia Tecnológica. [Internet]. 2004; (25). [Recuperado 30 diciembre 2019]. Disponible en: https://www.redalyc.org/articulo.oa?id=94402508

3. Ramírez H, Jaramillo J. Agentes naturales como alternativa para el tratamiento del agua. Rev Fac Ciencias Básicas. 2016;11(2):136. doi: 10.18359/rfcb.1303.

4. Feria J, Polo L, Hernández E. Evaluación de lodos de coagulación de agua cruda tratada con Moringa oleifera para uso agrícola. Ing e Investig. 2016;36(2):14-20.

5. Canett R, Arvayo K, Ruvalcaba N. Aspectos tóxicos más relevantes de Moringa oleifera y sus posibles daños. Rev Ciencias Biológicas y la Salud. 2014; XVI(2):36-43.

6. Sánchez Y, Martínez G, Sinagwa S, Vásquez J. Moringa oleifera; Importancia, Funcionalidad y Estudios Involucrados. Rev Científica la Univ Autónoma Coahuila. 2013; 5(9):25-30.

7. Rodríguez S, Muñoz R, García O, Fernández E. Empleo de un producto Coagulante Natural para Clarificar Agua. Rev CENIC Ciencias Quim [Internet]. 2005;36:8. [recuperado 16 diciembre 2019]. Disponible en: https://revista.cnic.edu.cu/revistaCQ/ sites/default/files/articulos/ CQ-2005-4-CQ-038.pdf

8. Cardoso C, Bergamasco R, Sala E, Konradt L. Otimização dos tempos de mistura e decantação no processo de coagulação / floculação da água bruta por meio da Moringa oleifera Lam. Acta Sci-Tecgnol. 2008;30(2):193-198.

9. Lédo P, Lima R, Paulo J, Duarte M. Estudio comparativo de sulfato de aluminio y semillas de Moringa oleifera para la depuración de aguas con baja turbiedad. Inf Tecnol. 2009;20(5):3-12.

10. Samia J. Uso apropiado de coagulantes naturales africanos para el abastecimiento de agua en el medio rural [Internet]. Lima, Perú: CEPIS/OPS/OMS; 1986. 427 p.

11. Martín C, Martín G, García A, Fernández T, Hernández E, Jürgen P. crítica Potential applications of Moringa oleifera. A critical review. Pastos y Forrajes. 2013;36(2):13749. 
12. Melo G, Turriago F. Evaluación de la eficiencia de la utilización de semillas de Moringa oleifera como una alternativa de biorremediacion en la purificación de aguas superficiales del caño cola de pato ubicado en el sector rural del municipio de acacias [Internet]. Villavicencio, Colombia: Universidad Nacional abierta y a distancia; 2012. [Recuperado 12 noviembre 2019], Disponible en: http://repository.unad.edu.co/handle/10596/1428

13. Oliveira NT, Nascimento KP, Gonçalves B de O, De Lima FC, André N. Tratamento de água com Moringa oleifera como coagulante/floculante natural. Revista Científica Da Faculdade De Educação E Meio Ambiente. 2018;9(1):373-382.

14. Gómez F, Salazar L. Evaluación de la eficiencia de semilla de (Moringa oleiera Lam.) como coagulante natural en la ciudad de Pasto-Colombia. Vitae. 2016;23:708-712.

15. Paniagua A, Chora J. Elaboración de aceite de semillas de Moringa oleifera para diferentes usos. Rev Ciencias la Salud. 2016;3(9):36-46.

16. Meza-Leones M, Riaños-Donado K, Mercado-Martínez I, Olivero-Verbel R, JuradoEraso M.Evaluación del poder coagulante del sulfato de aluminio y las semillas de Moringa oleifera en el proceso de clarificación del agua de la ciénaga de MalamboAtlántico. Revista UIS Ingenierías. 2018;17(2):95-103.

17. Aguirre SE, Piraneque NV. Sustancias naturales: alternativa para el tratamiento de agua. Inf Tecnol. 2018;29(3):59-70.

18. Fuentes N, Molina E, Ariza C. Coagulantes naturales en sistemas de flujo continuo, como sustituto del Al2(SO4)3 para clarificación de aguas. Prod + Limpia. [Internet]. 2016;11(2):41-54. [Recuperado 22 octubre 2019]. Disponible en: http://repository. lasallista.edu.co:8080/ ojs/index.php/pl/article/view/1238

19. Guzmán L, Taron A, Núñez A. Polvo de a semilla Cassia fistula como coagulante natural en el tratamiento de agua cruda. Biotecnoloía en el Sect Agropecu y Agroindustrial [Internet]. 2015;13(2):123. [Recuperado 16 noviembre 2019]. Disponible en: http:// revista biotecnologia.unicauca.edu.co/revista/index.php/biotecnologia/article/view/411 biotecnologia.unicauca.edu.co/revista/index.php/biotecnologia/article/view/411 